23

\title{
Spectrophotometric Determination of Carbimazole and Its Major Impurity, Degradation Product and Metabolite: Methimazole *
}

\author{
(C) S.S. Kurdaikar ${ }^{1}$, A. Fernandes ${ }^{1}$, S.V. Gandhi ${ }^{2}$, P. Pattewar ${ }^{3}$, and A.A. Mahajan ${ }^{1,9}$ \\ ${ }^{1}$ Goa College of Pharmacy, Panaji \\ 403001, Goa, India \\ ${ }^{2}$ AISSMS College of Pharmacy, Near RTO, \\ 411001 Pune, India \\ ${ }^{3}$ Slayback pharma, Kukatpally Hyderabad \\ 500072, India \\ "e-mail: anand_mahajan@yahoo.com
}

Received October 22, 2020

Revised October 22, 2021

Accepted Apri 02, 2021

The present research work was carried out in order to develop simple, accurate and precise UV sprctrophotometric methods having comparable sensitivity as that of sophisticated chromatographic techniques. Two methods were developed namely first derivative spectrophotometry and ratio spectra derivative spectrophotometry for accurate determination of specified impurity methimazole (imp A) in presence of drug carbimazole. First derivative spectrophotometric method involves recording of zero order spectra of both the drugs carbimazole and methimazole and its mixture in the range of $200-400 \mathrm{~nm}$ and subsequent conversion of these spectra into first derivative spectra. The drugs carbimazole and methimazole were determined by using zero crossing wavelengths of 227 and $260 \mathrm{~nm}$ respectively. In the second approach, ratio spectra were recorded for carbimazole and methimazole by selecting appropriate divisor concentration and converted into first derivative spectra. The determination of carbimazole and methimazole were carried out at wavelength 226.2 and $257 \mathrm{~nm}$, respectively. Both the methods were validated as per ICH guideline. The drugs carbimazole and methimazole showed linear response with good correlation coefficient and exhibited specificity, accuracy and precision within acceptable range. The second method of ratio spectra derivative spectrophotometry was found more sensitive as compare to first derivative spectrophotometry in detecting level of impurity methimazole up to $0.5 \%$ as per official specification. Hence, these developed methods can be used as alternative to sophisticated chromatographic technique for determination of assay and related impurity in bulk drug and formulation.

Keywords: carbimazole, methimazole, first derivative spectrophotometry, ratio spectra derivative spectrophotometry.

* Полный текст статьи опубликован в „Optics and Spectroscopy“ 2021 V. 129. N 7. 